OPEN ACCESS

Edited by:

Michael Kogut,

United States Department of

Agriculture, United States

Reviewed by:

Yulong Gao,

Harbin Veterinary Research Institute,

Chinese Academy of Agricultural

Sciences (CAAS), China

Shuang Chang,

Shandong Agricultural

University, China

*Correspondence:

Jianqiang Ye

jqye@yzu.edu.cn

Aijian Qin

aijian@yzu.edu.cn

tThese authors have contributed equally to this work

Specialty section:

This article was submitted to Veterinary Infectious Diseases,

a section of the journal

Frontiers in Veterinary Science

Received: 30 September 2021

Accepted: 25 October 2021

Published: 22 November 2021

Citation:

Mu Y, Xie Q, Wang W, Lu H, Lian M, Gao W, Li T, Wan Z, Shao H, Qin A and Ye J (2021) A Novel

Fiber-1-Edited and Highly Attenuated

Recombinant Serotype 4 Fowl

Adenovirus Confers Efficient

Protection Against Lethal Challenge.

Front. Vet. Sci. 8:759418.

doi: 10.3389/fvets.2021.759418

\section{A Novel Fiber-1-Edited and Highly Attenuated Recombinant Serotype 4 Fowl Adenovirus Confers Efficient Protection Against Lethal Challenge}

\author{
Yaru Mu ${ }^{1,2,3,4 \dagger}$, Quan Xie 1,2,3,4t, Weikang Wang ${ }^{1,2,3,4}$, Hao Lu ${ }^{1,2,3,4}$, Mingjun Lian ${ }^{1,2,3,4}$, \\ Wei Gao ${ }^{1,2,3,4}$, Tuofan Li $^{1,2,3,4}$, Zhimin Wan ${ }^{1,2,3,4}$, Hongxia Shao ${ }^{1,2,3,4}$, Aijian Qin ${ }^{1,2,3,4 *}$ and \\ Jianqiang $\mathrm{Ye}^{1,2,3,4 *}$
}

\begin{abstract}
${ }^{1}$ Ministry of Education Key Laboratory for Avian Preventive Medicine, Key Laboratory of Jiangsu Preventive Veterinary Medicine, Yangzhou University, Yangzhou, China, ${ }^{2}$ Jiangsu Co-innovation Center for Prevention and Control of Important Animal Infectious Diseases and Zoonoses, Yangzhou, China, ${ }^{3}$ Institutes of Agricultural Science and Technology Development, Yangzhou University, Yangzhou, China, ${ }^{4}$ Joint International Research Laboratory of Agriculture and Agri-Product Safety, the Ministry of Education of China, Yangzhou University, Yangzhou, China
\end{abstract}

Currently, a fatal disease of hepatitis-hydropericardium syndrome (HHS) caused by serotype 4 fowl adenovirus (FAdV-4) has spread worldwide and resulted in tremendous economic losses to the poultry industry. Various vaccines against FAdV-4 were developed to control the disease; however, few live-attenuated vaccines were available. In this study, we targeted the N-terminal of fiber-1 and rescued a recombinant virus FAdV4-RFP_F1 expressing the fusion protein of RFP and Fiber-1 based on the CRISPR/Cas 9 technique. In vitro studies showed that FAdV4-RFP_F1 replicated slower than the wild type FAdV-4, but the peak viral titer of FAdV4-RFP_F1 could still reach $10^{7.0} \mathrm{TCID}_{50} / \mathrm{ml}$ with high stability in LMH cells. Animal studies found that FAdV4-RFP_F1 not only was highly attenuated to the 2-week-old SPF chickens, but could also provide efficient protection against lethal challenge of FAdV-4. All these demonstrate that the recombinant virus FAdV4-RFP_F1 could be as an efficient live-attenuated vaccine candidate for FAdV-4, and the $\mathrm{N}$-terminal of fiber-1 could be as a potential insertion site for expressing foreign genes to develop FAdV-4-based vaccine.

Keywords: FAdV-4, fiber-1, CRISPR/Cas9, recombinant virus, attenuation, protection, vaccine

\section{INTRODUCTION}

Fowl adenovirus (FAdV) belongs to genus Aviadenovirus, family Adenoviridae (1). FAdV is a kind of non-enveloped viruses with a double-stranded DNA genome, clustered into 5 species (FAdV-A to FAdV-E) with 12 serotypes (FAdV-1 to FAdV-8a, FAdV-8b, and FAdV-9 to FAdV-11) based on the restriction enzyme digestion profile and sera cross-neutralization assay (2). FAdV can infect chickens through both vertical propagation and horizontal transmission. Of these 12 serotypes of FAdV, FAdV-4 is thought to be a highly pathogenic pathogen for poultry. The infection of FAdV-4 mainly results in hepatitis-hydropericardium syndrome (HHS) with a short latency, sudden onset, and high mortality. Recently, the disease caused by FAdV-4 has spread globally and lead to substantial economic losses to the poultry industry (3). Therefore, many vaccines or vaccine 
candidates including inactivated vaccines and sub-unit vaccines have been developed to control the disease (4). However, few live-attenuated vaccines against FAdV-4 were available.

It is well-known that the Fiber protein of FAdV plays a vital role in the infection and pathogenicity. Different from most serotypes of FAdV, FAdV-4 has two Fiber proteins, designated as Fiber-1 and Fiber-2 (5). Recently, Fiber-2 was identified to be closely related with the virulence of FAdV-4, and the fiber2-edited recombinant viruses FA4-EGFP and FAV-4_Del were highly attenuated to the SPF chickens (6-8). Notably, Fiber-1, but not Fiber-2, was identified to directly trigger viral infection via its knob and shaft domain through the cellular receptor, the CAR homology (9-11). To investigate whether the fiber-1 could be further edited to develop FAdV-4 live-attenuated vaccine or vector, we targeted the region between the tail and shaft of Fiber- 1 at the 87 th amino acid of its $\mathrm{N}$-terminus to generate a recombinant virus FAdV4-RFP_F1 with the expression of the fusion protein RFP (The Red Fluorescence Protein) and Fiber-1 through the CRISPR/Cas9 technique. In vitro and in vivo studies revealed that the rescued FAdV4-RFP_F1 was not only highly attenuated, but could also provide efficient protection against the lethal challenge.

\section{RESULTS}

\section{Generation of a Novel Recombinant Virus FAdV4-RFP_F1}

To explore whether the fiber- 1 could be edited to develop FAdV-4 live-attenuated vaccine or vector, two sgRNAs targeting the fiber-1 of FAdV-4 were designed and cloned into CRISPR/Cas9 vector, and subsequently transfected into LMH cells, followed with the transfection of the donor plasmid and the infection of FAdV-4 as shown in Figure 1A. After viral infection, RFP could be efficiently found in LMH cells transfected with sgRNA and donor plasmid, but no RFP could be found in LMH cells without infection (Figure 1B), indicating that the recombinant virus, designated as FAdV4-RFP_F1, was successfully generated. After purification by limiting dilution and plaque assay, the purified virus was confirmed by PCR, sequencing, and western blot. As described in Figure 1C, the specific single band of RFPfiber-1 could be amplified from the purified FAdV4-RFP_F1 whereas two bands could be amplified from the unpurified FAdV4-RFP_F1 by the specific primers listed in Table 2. Further identification with sequencing showed that the recombinant virus FAdV4-RFP_F1 was constructed exactly as designed (data
A
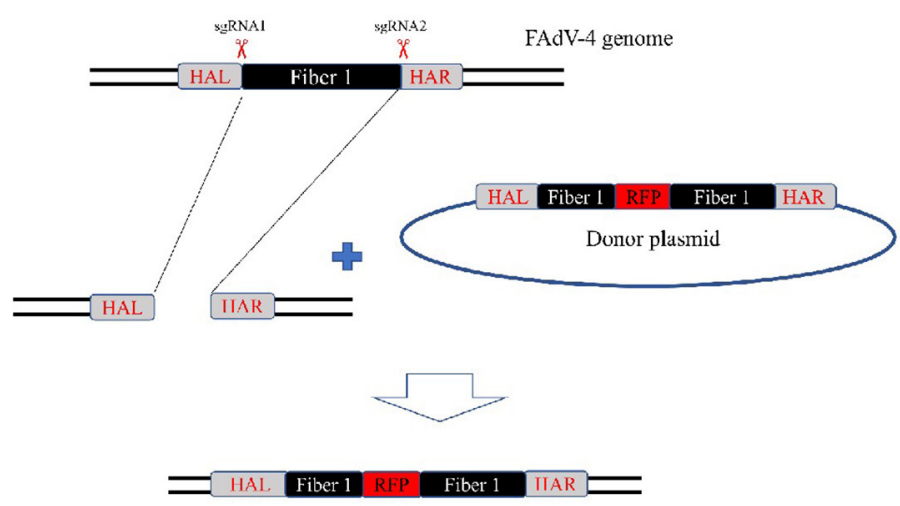

B

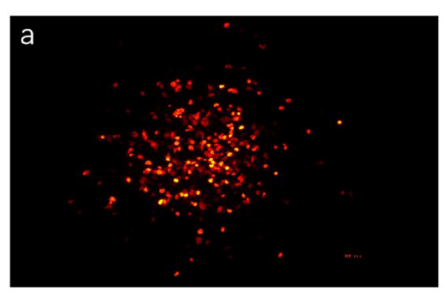

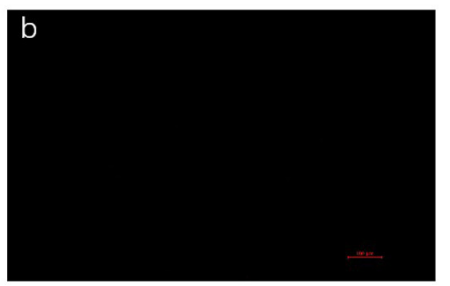

C

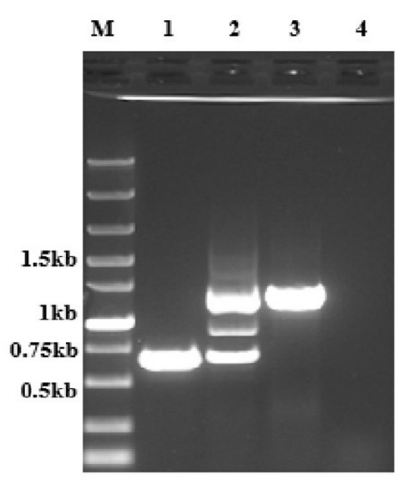

D

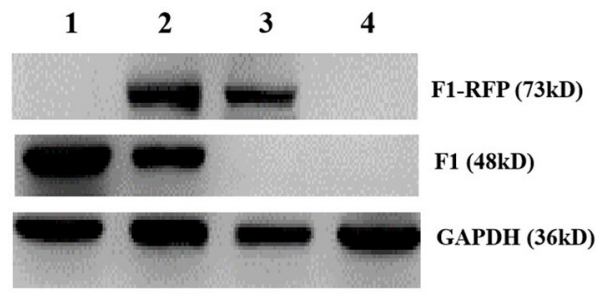

FIGURE 1 | Generation and identification of a novel recombinant virus FAdV4-RFP_F1. (A) Strategy of the CRISPR/Cas 9 system for generating the recombinant virus FAdV4-RFP_F1. LMH cells were transfected with sgRNA targeting the fiber-1 gene. At 24 hpi, the LMH cells were inoculated with FAdV-4 and transfected with donor plasmid. Then the recombinant virus FAdV4-RFP_F1 was purified by limiting dilution assay and viral plaque assay. (B) The RFP could be found by fluorescence microscopy in LMH cells transfected with sgRNA and donor plasmid (a), but not be found in LMH cells without infection (b). (C) PCR identification of the recombinant virus FAdV4-RFP_F1. The WT FAdV-4 (lane 1), unpurified recombinant virus FAdV4-RFP_F1 (lane 2), and the purified FAdV4-RFP_F1 (lane 3) were detected using specific primers. (D) Western blot analysis of the recombinant virus FAdV4-RFP_F1. The LMH cells infected with the WT FAdV-4 (lane 1). The unpurified recombinant FAdV4-RFP_F1 (lane 2) and the purified FAdV4-RFP_F1 (lane 3) were harvested and lysed, and the lysates were then tested with Western blot by chicken sera against FAdV-4 Fiber-1. 
not shown). In addition, western blot assay showed that the specific band of RFP-Fiber-1 fusion protein could be efficiently detected in the LMH cells infected with the purified FAdV4RFP_F1 as shown in Figure 1D. Taken together, all these data demonstrate that the recombinant virus FAdV4-RFP_F1 expressing RFP-Fiber-1 fusion protein is successfully generated.

\section{Efficient Replication and High Stability of FAdV4-RFP_F1 in vitro}

To investigate the growth characteristics of the recombinant virus in vitro, FAdV4-RFP_F1 and WT FAdV-4 were inoculated into $\mathrm{LMH}$ cells at the same dose and the viral supernatants were collected at different time points and determined by TCID $_{50}$.
As shown in Figure 2A, the recombinant virus FAdV4-RFP_F1 with fiber-1-edited could still replicate efficiently in LMH cells. Although the FAdV4-RFP_F1 replicated slower than the WT FAdV-4, the peak viral titer of FAdV4-RFP_F1 could still reach $10^{7.0} \mathrm{TCID}_{50} / \mathrm{ml}$ at $5 \mathrm{dpi}$. Moreover, to test the stability of the recombinant virus, the FAdV4-RFP_F1 was serially passaged with 15 generations in LMH cells, and then the viral supernatants were collected and examined by PCR. As shown in Figure 2B, the unique band of the RFP-fiber-1 could be efficiently amplified by the specific primers whereas the band specific to the WT FAdV4 could not be amplified in each generation of FAdV4-RFP_F1 tested. All these reveal that the FAdV4-RFP_F1 can efficiently and stably replicate in $\mathrm{LMH}$ cells.

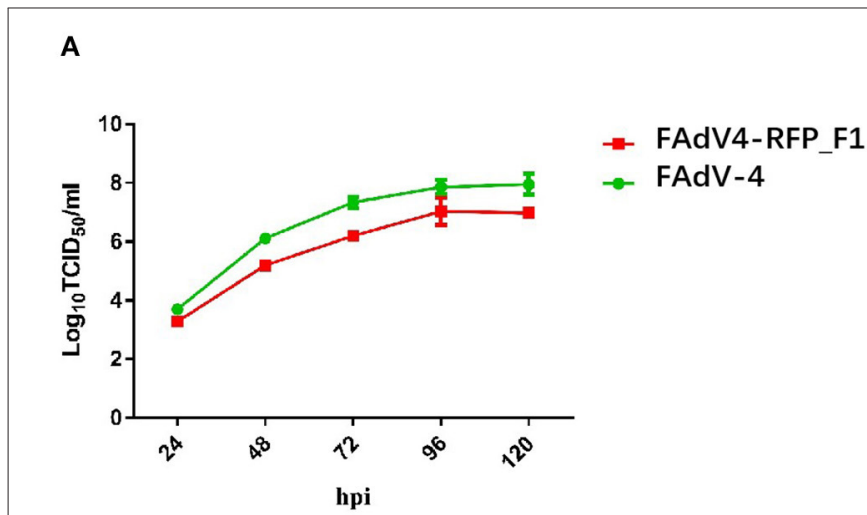

B

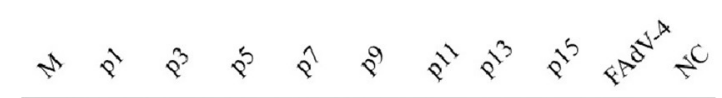

FIGURE 2 | Growth kinetics and stability of FAdV4-RFP_F1 in vitro. (A) LMH cells were inoculated with FAdV4-RFP_F1 and WT FAdV-4 at the same dose, then the virus-containing supernatants were collected at the indicated time points for virus titration. (B) The virus FAdV4-RFP_F1 was serially passaged for 15 generations with the dilution of 1:10,000 for each generation, and then the viral supernatants were collected and detected by PCR.

A

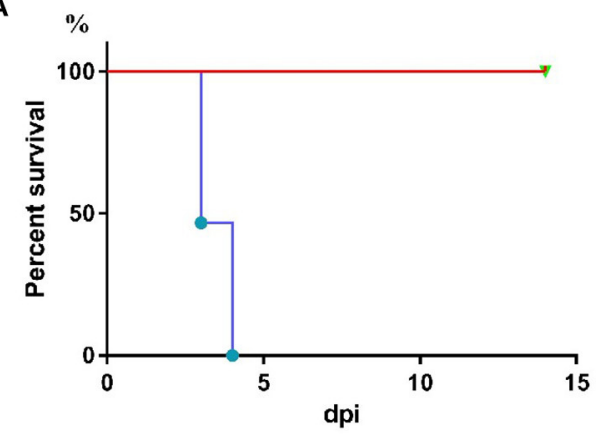

B

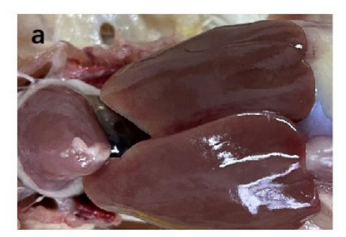

- FAdV4-RFP_F1

$\rightarrow$ NC

$\rightarrow$ FAdV-4

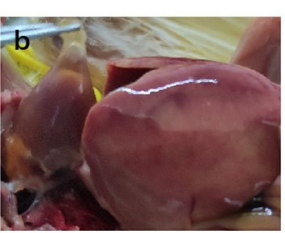

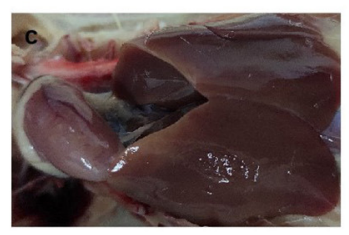

C

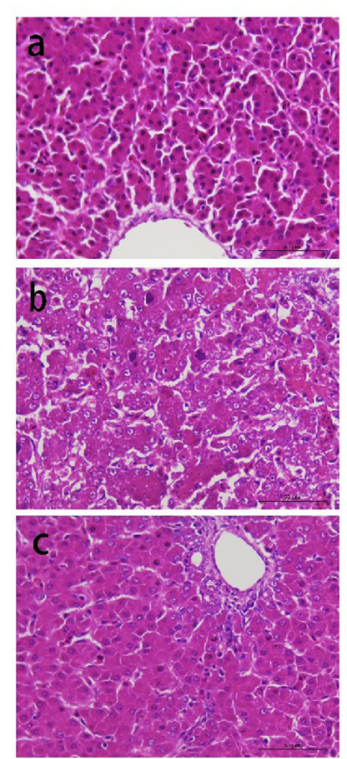

FIGURE 3 | FAdV4-RFP_F1 was highly attenuated in vivo. All the SPF chickens were randomly divided into three groups and inoculated with FAdV-4, FAdV4-RFP_F1, and $1 \%$ culture medium, respectively. All the chickens were monitored for 14 days. (A) Percent of survival for the infected chickens. (B) Pathological changes in the chickens from the chickens inoculated with FAdV4-RFP_F1 (a), FAdV-4 (b), and the negative control group (c). (C) Representative histological changes in liver tissues from the chickens inoculated with FAdV4-RFP_F1 (a), FAdV-4 (b), and the negative control group (c). 


\section{FAdV4-RFP_F1 Was Highly Attenuated in vivo}

To evaluate the replication and pathogenicity of the recombinant virus FAdV4-RFP_F1 in vivo, SPF chickens were infected with FAdV4-RFP_F1 and WT FAdV-4 virus at the same dose. The chickens inoculated with F12-DMEM containing 1\% of FBS were set as negative control. The clinical symptoms and mortality of the inoculated chickens were monitored daily. The cloacal swabs and organs from different groups were collected and analyzed at the indicated time points. The chickens infected with FAdV-4 exhibited signs of depression, loss of appetite, lethargy, and yellow-green and thin feces at 2 dpi. Fifty-three percent and $100 \%$ of the chickens died at $3 \mathrm{dpi}$ and $4 \mathrm{dpi}$, respectively. In contrast, chickens infected with FAdV4-RFP_F1 were all survived and did not show any clinical symptoms as described in Figure 3A. Moreover, the typical lesion, hepatitishydropericardium syndrome, could be observed in the chickens infected with FAdV-4 after necropsy analysis, while that in the other two groups could not be found (Figure 3B). In addition, the histopathological analysis demonstrated that chickens in the FAdV-4 group presented sever IBH in liver tissues, whereas these in FAdV4-RFP_F1 group were similar with the negative control group (Figure 3C). According to the viral shedding in the cloaca, the viral titers of the chickens infected with FAdV4 could reach up to $10^{5} \mathrm{TCID}_{50} / \mathrm{ml}$ at $2-5 \mathrm{dpi}$, whereas no virus could be detected in the chickens infected with FAdV4RFP_F1 during 2-8 dpi (Figure 4A). For the virus tissue load, the high level of viral titer could be detected in the livers, spleens, and kidneys in the chickens infected with FAdV4 at 2-4 dpi. Of note, the viral titer in the livers in the chickens infected with FAdV-4 could reach up to $10^{8} \mathrm{TCID}_{50} / \mathrm{ml}$, followed by kidney and spleen, whereas no virus could be detected in the chickens infected with FAdV4-RFP_F1 by TCID $_{50}$ as shown in Figures 4B-D. However, the recombinant virus FAdV4-RFP_F1 in the organic tissues could be detected by PCR at early time points (Figure 4E). Taken together, all these demonstrate that the FAdV4-RFP_F1 is highly attenuated in vivo.
A

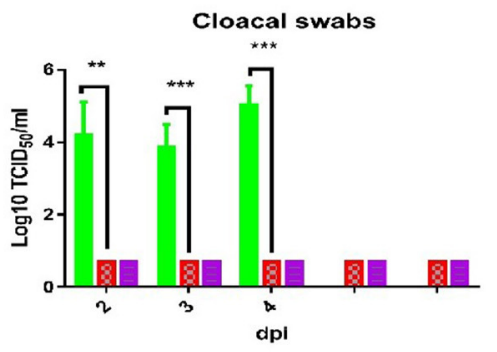

C

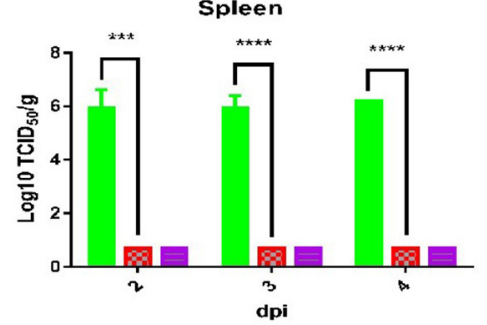

E

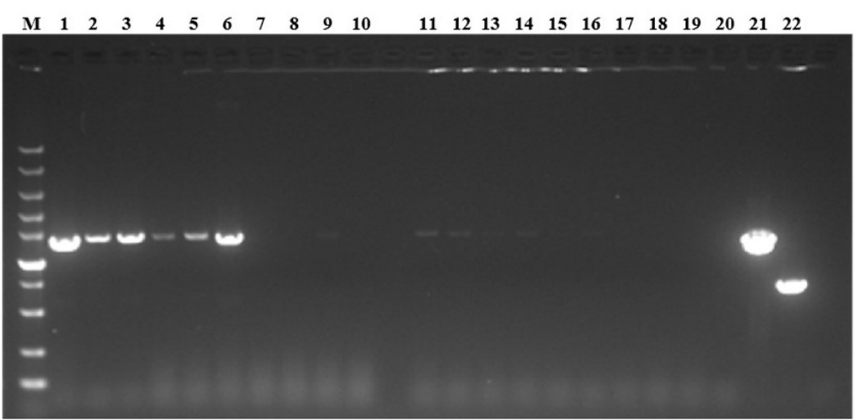

B

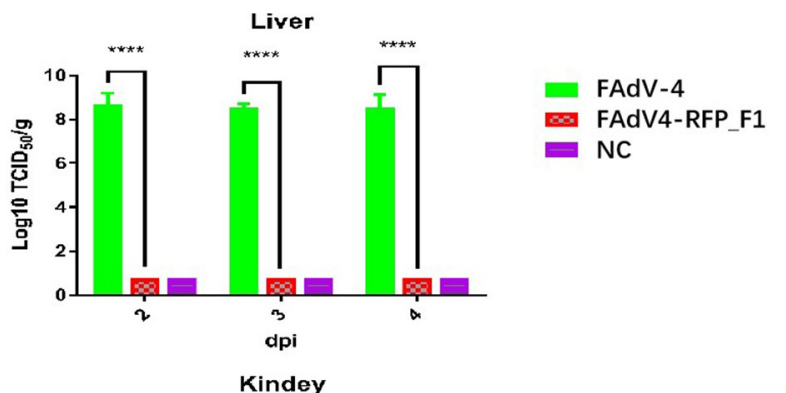

D

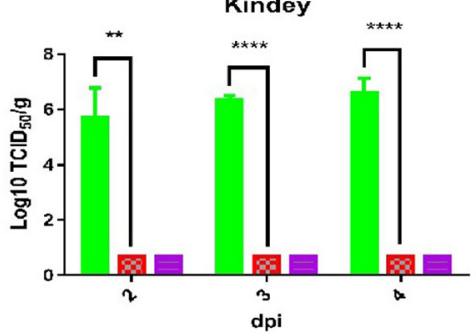

FAdV-4
FAdV4-RFP_F1
NC

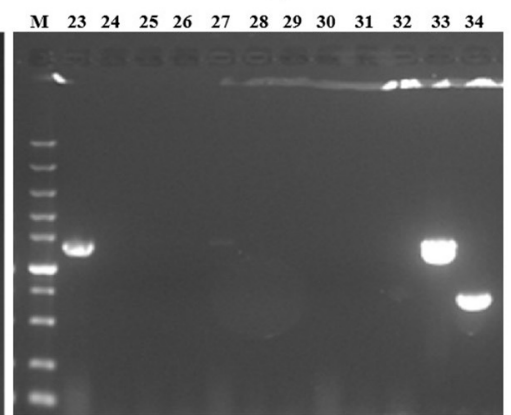

FIGURE 4 | Viralsheddingin cloaca andload in tissues from the infected chickens. (A) Viral shedding in cloacal swabs from the infected chickens. Viral loads in liver (B), spleen (C), and kidney (D) from the infected chickens. (E) PCR identification of tissues from the chickens infected with FAdV4-RFP_F1. Liver samples (2 dpi: lane 1-3; 3 dpi: lane 4-6; 4 dpi: lane 7-9); spleen samples (2 dpi: lane 11-13; 3 dpi: lane14-16; 4 dpi: lane 17-19); kidney samples (2 dpi: lane 23-25; 3 dpi: lane 26-28; 4 dpi: lane 29-31). PCR identification of tissues from the chickens of the negative control group (liver sample: lane 10; spleen sample: lane 20; kidney sample: lane 32). Cell supernatant of FAdV4-RFP_F1 (lane 21 and 33); cell supernatant of FAdV-4 (lane 22 and 34). ${ }^{* *},{ }^{* \star *}$, and ${ }^{* \star * *}$ indicate $P$-value $<0.05,0.01,0.001$, and 0.0001 , respectively. 


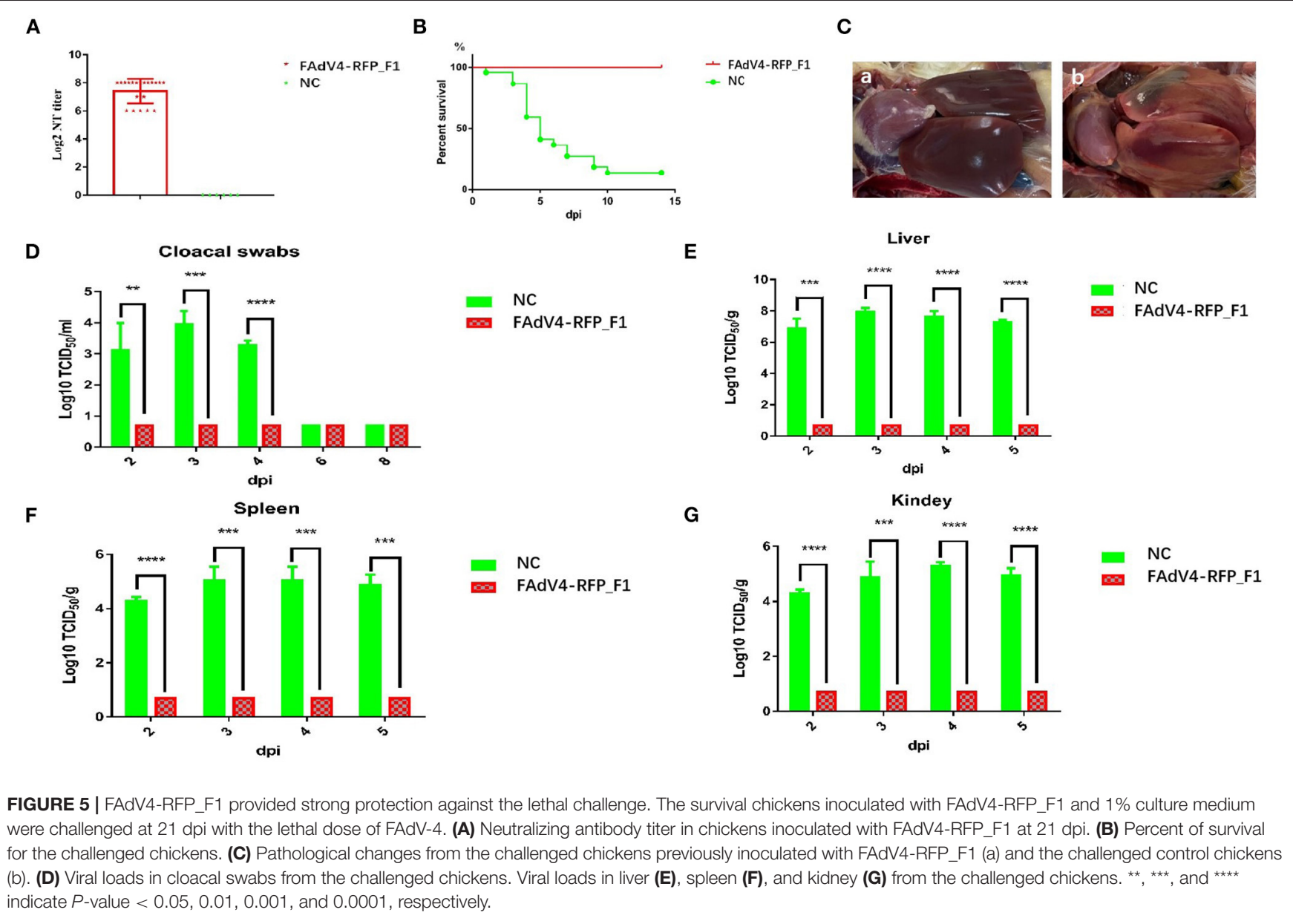

\section{FAdV4-RFP_F1 Provided Efficient Protection Against Lethal Challenge}

To evaluate whether the recombinant virus FAdV4-RFP_F1 could provide protective efficacy against FAdV-4, the sera from chickens in the control group or infected with FAdV4-RFP_F1 were first collected at $21 \mathrm{dpi}$ for the detection of neutralizing antibody, and all these chickens were then challenged with the lethal dose of the WT FAdV-4 at $21 \mathrm{dpi}$. The clinical symptoms and mortality of the chickens were monitored daily. The cloacal swab and tissue samples from chickens were collected at the indicated time points and titrated for viral titers. As described in Figure 5A, the chickens infected with FAdV4-RFP_F1 at 21 dpi could produce high level of neutralizing antibody with the average titer of about $2^{7.4}$ whereas the neutralizing antibody could not be detected in the control group. After challenge, the chickens in the control group showed clinical symptom and the mortality rate reached $82 \%(19 / 23)$ at $10 \mathrm{dpi}$, whereas the chickens previously infected with FAdV4-RFP_F1 did not show any clinical symptoms or death through the animal experiment as shown in Figure 5B. In the necropsy analysis, the typical lesion, hepatitis-hydropericardium syndrome was observed in the chickens from the control group (Figure 5C). Moreover, the high level of viral titers in the cloacal swab and tissue samples from the chickens of the control group were efficiently detected, whereas those from chickens previously infected with FAdV4RFP_F1 could be not detected (Figures 5D-G). All these data clearly demonstrate that the recombinant virus FAdV4-RFP_F1 can provide efficient protection against the lethal challenge of FAdV-4.

\section{DISCUSSION}

Hepatitis-hydropericardium syndrome caused by the highly pathogenic FAdV-4 has resulted in substantial economic losses to poultry industry globally. To control the disease, the vaccination is one of the efficient strategies (12). Up to now, various vaccines or vaccine candidates have been generated, including the inactivated vaccines, the recombinant subunit vaccines, and the live-attenuated vaccines (4, 13-17). Generally, different vaccines have different advantages or disadvantages. The inactivated whole virus vaccines can provide efficient protection, but these vaccines are generally costly. Although the Fiber-2 subunit vaccines are also widely used, Fiber- 2 cannot induce detectable neutralizing antibodies to completely prevent the viral shedding. The live-attenuated vaccines can both induce strong humoral and cellular immune responses and show a great promise for efficiently controlling the diseases caused by 
FAdV-4. Currently, the live-attenuated vaccines against FAdV4 were mainly generated through cell or host adaptation of the virulent strains (4). Since the altered or adapted genomes of such live-attenuated vaccines are generally uncontrolled, liveattenuated vaccines against FAdV-4 with specific gene-edited are badly needed.

Recently, we used CRISPR-Cas9 technique to target the virulent gene fiber-2 to generate two live-attenuated fiber-2edited vaccine candidates FA4-EGFP and FAV-4_Del $(7,8)$. In this study, we targeted another structural protein Fiber-1 of FAdV-4 to rescue a novel recombinant virus FAdV4-RFP_F1 expressing the fusion protein of RFP and Fiber-1. Since our previous study demonstrated that the shaft and knob domains of Fiber-1 play vital roles in directly triggering the viral infection of FAdV-4 (10), the inserted site of RFP and the sgRNA target were designed at the region between the tail and shaft of Fiber1 at the 87th amino acid of its $\mathrm{N}$-terminus. Of note, the rescued FAdV4-RFP_F1 not only replicated efficiently and stably in vitro, but was also avirulent in vivo. These indicate that although the Fiber-1 plays a critical role in triggering the infection of FAdV-4, the N-terminal domain of Fiber-1 can be an efficient target to be edited to generate recombinant viruses for expressing foreign genes. Moreover, the recombinant virus FAdV4-RFP_F1 did not shed in the cloaca and showed limited replication in the tissues tested in the inoculated chickens, but it could provide efficient protection against the lethal challenge of the WT FAdV-4, highlighting FAdV4-RFP_F1 generated here can be as an efficient live-attenuated vaccine for preventing the diseases caused by FAdV-4.

It should be noted that although the recombinant virus FAdV4-RFP_F1 could replicate efficiently in LMH cells, but it could not efficiently replicate in liver, spleen, and kidney in the infected chickens through intramuscular injection. Whether the recombinant virus FAdV4-RFP_F1 with the edited fiber1 can alter the tissue tropism in vivo needs to be elucidated. Recently, although Zhang et al. showed that the knob of Fiber-1 could also be edited to insert the exogenous peptide, several generated recombinant viruses could alter the cell tropism in vitro (18). To investigate the mechanism for the protective efficacy for FAdV4-RFP_F1 against FAdV-4, the sera from chickens before challenge were collected and tested for neutralizing antibody. It was unexpected that these chickens inoculated with FAdV4-RFP_F1 at 21 dpi could produce high level of neutralizing antibody with the average titer of about $2^{7.4}$ (Figure 5A). Wu et al. found that the expression of the anti-inflammatory cytokines IL-4, IL-10, and transforming growth factor (TGF)- $\beta 1$ and TGF- $\beta 2$ were upregulated in FAdV-4-infected chickens (19) and the infection of FAdV-4 could induce strong innate immune responses in chickens (20). Therefore, except for the contribution of the humoral immune response, the innate and cellular immune response induced by FAdV4-RFP_F1 in the inoculated chickens should play vital roles in the protection, which need to be further clarified.

In summary, this is the first demonstration of a novel fiber-1-edited and highly attenuated recombinant virus FAdV4RFP_F1. Notably, FAdV4-RFP_F1 could efficiently replicate in vitro, but not in vivo. However, FAdV4-RFP_F1 could provide strong protection against the lethal challenge of FAdV4. The generation of the avirulent recombinant virus FAdV4RFP_F1 carrying the fusion protein of RFP and Fiber-1 with high viral titer highlights FAdV4-RFP_F1 can be an efficient live-attenuated vaccine against FAdV-4, and the N-terminal domain of fiber-1 can be edited as a potential insertion site for expressing foreign genes. The cellular immune protection induced by the live-attenuated recombinant virus FAdV4RFP_F1 needs to be further elucidated. Since the co-infection could significantly promote the pathogenicity of FAdV-4 and inhibit the immune response in chickens (21), whether the RFP in FAdV4-RFP_F1 can be replaced with other protective antigens to develop FAdV-4 based multiple vaccines both for controlling of FAdV-4 and other poultry pathogens also need to be evaluated.

\section{MATERIALS AND METHODS}

\section{Cells, Viruses, and Antibodies}

Chicken hepatoma cells (Leghorn male hepatoma, LMH) were purchased from American Type Culture Collection (Manassas, VA, USA) and cultured in F12-Dulbecco's Modified Eagle Medium (DMEM; Gibco, NY, USA) supplemented with $10 \%$ fetal bovine serum (FBS; Lonsera, Shanghai, China) in a 5\% $\mathrm{CO}_{2}$ incubator at $37^{\circ} \mathrm{C}$, and passaged every 3 days. The FAdV4 strain SD2015 was isolated and stored in our laboratory. The monoclonal antibody (mAb) 3C2 against Fiber-2 of FAdV4 and chicken sera against FAdV-4 were prepared in our laboratory (22).

\section{Construction of sgRNA and Donor Plasmids}

The sgRNA targeting the fiber-1 of FAdV-4 was designed according to the CRISPR guide RNA designing website (www.benchling.com) and cloned into the CRISPR/Cas9 vector lentiCRISPR v2 (23). The sequences of the sgRNA were listed in Table 1. The donor plasmid with the RFP gene located after the N-terminal 87 amino acid of the fiber-1 was constructed by overlap extension PCR and the homologous arm (HA) was designed in 1,000 bp length at both ends, respectively. The template was assembled as the HAL-F1-RFPF1-HAR and then cloned into the pMD19 simple vector. The primers used for constructing donor plasmid were shown in Table 2.

TABLE 1 | Primers used for the construction of sgRNA.

\begin{tabular}{ll}
\hline Sequence of primers $\left(\mathbf{5}^{\prime} \boldsymbol{\rightarrow} \mathbf{3} \mathbf{3}^{\prime}\right)$ \\
\hline sgRNA1 & F1-sgRNA1_F: CACCGACCTAGCCGGTACCTITCGG \\
& F1-sgRNA1_R: AAACCCGAAAGGTACCGGCTAGGTC \\
sgRNA2 F1-sgRNA2_F: CACCGGGTTACGTCTACTCCCCCAA & F1-sgRNA2_R: AAACTTGGGGGAGTAGACGTAACCC
\end{tabular}


TABLE 2 | Primers used for the construction of donor plasmid and the identification of the recombinant virus.

\begin{tabular}{ll}
\hline PCR products & Sequence of primers $\left(\mathbf{5}^{\prime} \rightarrow \mathbf{3}^{\prime}\right)$ \\
\hline RFP & F: CCCATCGGGGTCGACCGCGATCTG \\
& R: CCCATCGGGGTCGACCGCGATCTG \\
HAL+Fiber-1 & F: GACCGGGGACATTATACTGTC \\
Fiber-1+HAR & R: CCCATCGGGGTCGACCGCGATCTG \\
& F: CCCATCGGGGTCGACCGCGATCTG \\
Partial Fiber-1 & R: CCCATCGGGGTCGACCGCGATCTG \\
& F: CTGACGGTAATCGGTCTCGG \\
& R: CTGTGAGTCGACGAGCACTT
\end{tabular}

\section{Rescuing of the Recombinant Virus FAdV4-RFP_F1}

LMH cells seeded in 6-well plate were transfected with sgRNAs targeting the fiber-1 gene at $2 \mu \mathrm{g}$ of each. At 24-h posttransfection (hpt), the LMH cells were inoculated with FAdV4 at a multiplicity of infection (MOI) of 0.1 and immediately transfected with the donor plasmid at $4 \mu \mathrm{g}$. The rescued recombinant virus, designated as FAdV4-RFP_F1, was observed through a fluorescence microscopy and further purified by limiting the dilution assay and virus plaque assay. The purified virus was then identified by PCR, sequencing, and western blot.

\section{Growth Curve of the Recombinant Virus FAdV4-RFP_F1}

The recombinant virus FAdV4-RFP_F1 and wild type FAdV4 were inoculated into LMH cells at $0.1 \mathrm{MOI}$, respectively. At $2 \mathrm{~h}$ post-infection (hpi), the cells were washed with PBS and maintained with the fresh F12-DMEM containing 1\% of FBS. Then, the viral supernatants were harvested at $24,48,72,96$, and $120 \mathrm{dpi}$, and then titrated in LMH cells by IFA. The TCID 50 of the viruses were calculated by the Reed-Muench method (24). The final viral growth curves were constructed with GraphPad Prism 7 software.

\section{Stability of the Recombinant Virus FAdV4-RFP_F1}

To test the stability, the purified recombinant virus was serially passaged in LMH cell with the dilution of 1:10,000 for each generation, and the supernatants were collected from each generation and examined by PCR using specific primers.

\section{Western Blot Assay}

LMH cells either infected with the recombinant virus FAdV4-RFP_F1 or wild type FAdV-4 were lysed in lysis buffer (CST, MA, USA) with phenylmethylsulfonyl fluoride (PMSF; Beyotime, Shanghai, China), protease, and phosphatase inhibitors (CST, MA, USA). The lysates were then boiled in the loading buffer. After subjected to $10 \%$ SDS-PAGE, the denatured proteins were transferred to nitrocellulose (NC) membranes (GE Healthcare Life sciences, Freiburg, Germany). After blocking with 5\% skimmed milk in PBS with Tween 20 (PBST) for $2 \mathrm{~h}$ at room temperature (RT), the membranes were reacted with the corresponding mAbs or sera for $2 \mathrm{~h}$ at RT. After three washes with PBST, the membranes were incubated with horseradish peroxidase (HRP)-labeled secondary antibodies for $1 \mathrm{~h}$ at RT. After another three washes, the membranes were developed with chemiluminescent reagents and imaged with an automatic imaging system (Tanon 5200).

\section{Indirect Immunofluorescent Assay}

The LMH cells infected with viruses were fixed with the icecold acetone-ethanol (3:2) mixture for $5 \mathrm{~min}$ at RT and washed with PBS. The fixed cells were then incubated with the diluted $\mathrm{mAb} 3 \mathrm{C} 2$ against Fiber-2 for $45 \mathrm{~min}$ at $37^{\circ} \mathrm{C}$. After washing three times with PBS, the cells were incubated with the diluted secondary antibody (goat anti-mouse antibody labeled with fluorescein isothiocyanate, FITC) for another $45 \mathrm{~min}$ at $37^{\circ} \mathrm{C}$. Again, after three washes with PBS, the cells were observed by invert fluorescence microscopy.

\section{Neutralization Test}

Different dilutions of the chicken sera were first mixed with $200 \mathrm{TCID}_{50}$ of FAdV-4 and incubated for $1 \mathrm{~h}$ at $37^{\circ} \mathrm{C}$. Then, the mixtures were added to the 96-well plate with $\mathrm{LMH}$ cells and incubated for $2 \mathrm{~h}$ at $37^{\circ} \mathrm{C}$. After washing once, the cells were cultured in F12-DMEM with $1 \%$ fetal bovine serum. After culturing for $96 \mathrm{~h}$, the cells were fixed and subjected to IFA analysis by using $\mathrm{mAb} 3 \mathrm{C} 2$ against Fiber-2 as previously described (22).

\section{Animal Experiment}

A total of 102 2-week-old specific pathogen free (SPF) chickens were randomly divided into three groups (chickens in group I $(n=34)$ were infected with FAdV-4 as a positive control group; chickens in group II $(n=34)$ were infected with FAdV4-RFP_F1 as experimental group; and chickens in group III $(n=34)$ without infection was set as negative control) and inoculated through intramuscular injection. The chickens in group I and II were inoculated at a dose of $2 \times 10^{5}$ $\mathrm{TCID}_{50}$ in $200 \mu \mathrm{l}$ of $1 \%$ culture medium, whereas the chickens in group III were inoculated with the same volume of $1 \%$ culture medium. The cloacal swabs of each group were collected at indicated time points post-infection. Three chickens in each group were euthanized at 2, 3, and $4 \mathrm{dpi}$. The livers, spleens, and kidneys of these chickens were collected for viral titration or histopathological analysis. At $21 \mathrm{dpi}$, chickens that survived in group II and group III were challenged with 2 $\times 10^{6}$ TCID $_{50}$ of FAdV-4 in $200 \mu \mathrm{l}$ of $1 \%$ culture medium via intramuscular injection to further evaluate the protective efficacy. The cloacal swabs were collected at indicated time points post-challenge $(\mathrm{pc})$. Three chickens in each group were euthanized at 2, 3, 4, and $5 \mathrm{dpc}$. The livers, spleens, and kidneys of these chickens were collected for viral titration. The 
clinical symptoms and mortality of the inoculated chickens were monitored daily.

\section{Titration of Viral Titer in the Organs and Cloacal Swab Samples}

The homogenates of liver, spleen, and kidney from the chickens were treated with $10 \times$ penicillin and streptomycin for $1 \mathrm{~h}$ at $37^{\circ} \mathrm{C}$ and centrifuged to obtain the supernatant. The cloacal samples collected from the chickens were placed in $800 \mu \mathrm{l}$ of PBS. After three times of repeated freeze-thaw cycles, the samples were also treated with $10 \times$ penicillin and streptomycin for $1 \mathrm{~h}$ at $37^{\circ} \mathrm{C}$ and centrifuged to obtain the supernatant. The virus-containing supernatants were serially diluted in F12DMEM with $1 \%$ of FBS and were inoculated into the 96well plates with LMH cells. The infected LMH cells were then fixed and detected by IFA using mAb 3C2 against Fiber- 2 at 4 dpi, and $\mathrm{TCID}_{50}$ of those supernatants were calculated by the Reed-Muench method.

\section{Statistical Analysis}

All the results are presented as means \pm standard deviation. The statistical analysis in this study was performed with the Student's $t$-test using GraphPad 7 software. $P$-value of $<0.05$ was considered significant. ${ }^{*}, * *, * *$, and ${ }^{* * *}$ indicate $P$-value $<0.05$, $0.01,0.001$, and 0.0001 , respectively.

\section{DATA AVAILABILITY STATEMENT}

The original contributions presented in the study are included in the article/supplementary material, further inquiries can be directed to the corresponding author/s.

\section{REFERENCES}

1. Oksanen HM, Bamford DH. Family tectiviridae. In: King AMQ, Adams MJ, Carstens EB, Lefkowitz EJ, editors. Virus Taxonomy, Ninth Report of the International Committee on Taxonomy of Viruses. Amsterdam: Elsevier/Academic Press. (2012).

2. Hess M. Detection and differentiation of avian adenoviruses: a review. Avian Pathol. (2000) 29:195-206. doi: 10.1080/03079450050 045440

3. Ye J, Liang G, Zhang J, Wang W, Song N, Wang P, et al. Outbreaks of serotype 4 fowl adenovirus with novel genotype, China. Emerg Microbes Infect. (2016) 5:e50. doi: 10.1038/emi.2016.50

4. Schachner A, Matos M, Grafl B, Hess M. Fowl adenovirus-induced diseases and strategies for their control-a review on the current global situation. Avian Pathol. (2018) 47:111-26. doi: 10.1080/03079457.2017.1385724

5. Marek A, Nolte V, Schachner A, Berger E, Schlotterer C, Hess M. Two fiber genes of nearly equal lengths are a common and distinctive feature of Fowl adenovirus C members. Vet Microbiol. (2012) 156:411-7. doi: 10.1016/j.vetmic.2011.11.003

6. Zhang Y, Liu R, Tian K, Wang Z, Yang X, Gao D, et al. Fiber2 and hexon genes are closely associated with the virulence of the emerging and highly pathogenic fowl adenovirus 4. Emerg Microbes Infect. (2018) 7:199. doi: 10.1038/s41426-018-0203-1

7. Xie Q, Cao S, Zhang W, Wang W, Li L, Kan Q, et al. A novel fiber2-edited live attenuated vaccine candidate against the highly pathogenic serotype 4 fowl adenovirus. Vet Res. (2021) 52:35. doi: 10.1186/s13567-021$00907-z$

\section{ETHICS STATEMENT}

The animal study was reviewed and approved by the Animal Care Committee at Yangzhou University in China.

\section{AUTHOR CONTRIBUTIONS}

JY and AQ designed the project. YM, QX, WW, HL, ML, ZW, and TL carried out the experiment. YM, QX, HS, ZW, and WG analyzed the data. YM, QX, and JY drafted the manuscript. JY supervised all the experiments and participated in the data analysis. YM, QX, ZW, HS, and AQ discussed and prepared the final reported. All of the authors have read and approved the final manuscript.

\section{FUNDING}

This study was supported by Jiangsu Agricultural Science and Technology Innovation Fund (CX(19)3026), the Key Research \& Development (R\&D) Plan in Yangzhou City (YZ2020052), Key Laboratory of Prevention and Control of Biological Hazard Factors (Animal Origin) for Agrifood Safety and Quality (26116120), and Research Foundation for Talented Scholars in Yangzhou University and the Priority Academic Program Development of Jiangsu Higher Education Institutions.

\section{ACKNOWLEDGMENTS}

We thank for Jianjun Zhang (Sinopharm Yangzhou VAC Biological Engineering Co. Ltd.) for kindly providing us SPF chickens.

8. Xie Q, Wang W, Li L, Kan Q, Fu H, Geng T, et al. Domain in Fiber-2 interacted with KPNA3/4 significantly affects the replication and pathogenicity of the highly pathogenic FAdV-4. Virulence. (2021) 12:754-65. doi: 10.1080/21505594.2021.1888458

9. Pan Q, Wang J, Gao Y, Wang Q, Cui H, Liu C, et al. Identification of chicken CAR homology as a cellular receptor for the emerging highly pathogenic fowl adenovirus 4 via unique binding mechanism. Emerg Microbes Infect. (2020) 9:586-96. doi: 10.1080/22221751.2020.1736954

10. Wang W, Liu Q, Li T, Geng T, Chen H, Xie Q, et al. Fiber-1, not fiber-2, directly mediates the infection of the pathogenic serotype 4 fowl adenovirus via its shaft and knob domains. J Virol. (2020) 94:e00954-20. doi: 10.1128/JVI.00954-20

11. Zou X, Rong Y, Guo X, Hou W, Lu Z. Fiber1, but not fiber2, is the essential fiber gene for fowl adenovirus 4 (FAdV-4). J Gen Virol. (2021) 102:1-12. doi: 10.1099/jgv.0.001559

12. Wang K, Sun H, Li Y, Yang Z, Ye J, Chen H. Characterization and pathogenicity of fowl adenovirus serotype 4 isolated from eastern China. BMC Vet Res. (2019) 15:373. doi: 10.1186/s12917-019-2092-5

13. Kim MS, Lim TH, Lee DH, Youn HN, Yuk SS, Kim BY, et al. An inactivated oil-emulsion fowl Adenovirus serotype 4 vaccine provides broad crossprotection against various serotypes of fowl Adenovirus. Vaccine. (2014) 32:3564-8. doi: 10.1016/j.vaccine.2014.03.015

14. Meng K, Yuan X, Yu J, Zhang Y, Ai W, Wang Y. Identification, pathogenicity of novel fowl adenovirus serotype 4 SDJN0105 in Shandong, China and immunoprotective evaluation of the newly developed inactivated oil-emulsion FAdV-4 vaccine. Viruses. (2019) 11:627. doi: 10.3390/v110 70627 
15. Schachner A, Marek A, Jaskulsk B, Bilic I, Hess M. Recombinant FAdV4 fiber-2 protein protects chickens against hepatitis-hydropericardium syndrome (HHS). Vaccine. (2014) 32:1086-92. doi: 10.1016/j.vaccine.2013. 12.056

16. Shah MS, Ashraf A, Rahman M, Khan MI, Qureshi JA. A subunit vaccine against hydropericardium syndrome using adenovirus penton capsid protein. Vaccine. (2012) 30:7153-6. doi: 10.1016/j.vaccine.2012. 10.013

17. Wang $\mathrm{X}$, Tang Q, Chu Z, Wang $\mathrm{P}$, Luo $\mathrm{C}$, Zhang $\mathrm{Y}$, et al. Immune protection efficacy of FAdV-4 surface proteins fiber-1, fiber-2, hexon and penton base. Virus Res. (2018) 245:1-6. doi: 10.1016/j.virusres.2017. 12.003

18. Zhang W, Guo X, Yin F, Zou X, Hou W, Lu Z. Fiber modifications enable fowl adenovirus 4 vectors to transduce human cells. J Gene Med. (2021) 23:e3368. doi: 10.1002/jgm.3368

19. Wu N, Yang B, Wen B, Li W, Guo J, Qi X, et al. Pathogenicity and immune responses in specific-pathogen-free chickens during fowl adenovirus serotype 4 infection. Avian Dis. (2020) 64:315-23. doi: 10.1637/aviandiseases-D-20-00004

20. Zhao W, Li X, Li H, Han Z, Wang F, Liu C, et al. Fowl adenoviruse4 infection induces strong innate immune responses in chicken. Comp Immunol Microbiol Infect Dis. (2020) 68:101404. doi: 10.1016/j.cimid.2019. 101404

21. Xu A, Sun L, Tu K, Teng Q, Xue J, Zhang G. Experimental co-infection of variant infectious bursal disease virus and fowl adenovirus serotype 4 increases mortality and reduces immune response in chickens. Vet Res. (2021) 52:61. doi: 10.1186/s13567-02100932-y
22. Wang P, Zhang J, Wang W, Li T, Liang G, Shao H, et al. A novel monoclonal antibody efficiently blocks the infection of serotype 4 fowl adenovirus by targeting fiber-2. Vet Res. (2018) 49:29. doi: 10.1186/s13567-018-0525-y

23. Ran FA, Hsu PD, Wright J, Agarwala V, Scott DA, Zhang F. Genome engineering using the CRISPR-Cas9 system. Nat Protoc. (2013) 8:2281. doi: $10.1038 /$ nprot.2013.143

24. Reed LJ, Muench H. A simple method for estimating 50 percent endpoints. Am J Hyg. (1938) 27:493-7. doi: 10.1093/oxfordjournals.aje.a118408

Conflict of Interest: The authors declare that the research was conducted in the absence of any commercial or financial relationships that could be construed as a potential conflict of interest.

Publisher's Note: All claims expressed in this article are solely those of the authors and do not necessarily represent those of their affiliated organizations, or those of the publisher, the editors and the reviewers. Any product that may be evaluated in this article, or claim that may be made by its manufacturer, is not guaranteed or endorsed by the publisher.

Copyright (c) $2021 \mathrm{Mu}$, Xie, Wang, Lu, Lian, Gao, Li, Wan, Shao, Qin and Ye. This is an open-access article distributed under the terms of the Creative Commons Attribution License (CC BY). The use, distribution or reproduction in other forums is permitted, provided the original author(s) and the copyright owner(s) are credited and that the original publication in this journal is cited, in accordance with accepted academic practice. No use, distribution or reproduction is permitted which does not comply with these terms. 